\title{
Patient Position Detection for SAR Optimization in Magnetic Resonance Imaging
}

\author{
Andreas Keil ${ }^{1,3}$, Christian Wachinger ${ }^{1}$, Gerhard Brinker ${ }^{2}$, Stefan Thesen $^{2}$, \\ and Nassir Navab ${ }^{1}$ \\ ${ }^{1}$ Chair for Computer Aided Medical Procedures (CAMP), TU Munich, Germany \\ \{keila, wachinge, navab\}@cs.tum.edu \\ ${ }^{2}$ Siemens Medical Solutions, Erlangen, Germany \\ ${ }^{3}$ Chirurgische Klinik und Poliklinik, Klinikum Innenstadt, Munich, Germany
}

\begin{abstract}
Although magnetic resonance imaging is considered to be non-invasive, there is at least one effect on the patient which has to be monitored: The heating which is generated by absorbed radio frequency (RF) power. It is described using the specific absorption rate (SAR). In order to obey legal limits for these SAR values, the scanner's duty cycle has to be adjusted. The limiting factor depends on the patient's position with respect to the scanner. Detection of this position allows a better adjustment of the RF power resulting in an improved scan performance and image quality. In this paper, we propose real-time methods for accurately detecting the patient's position with respect to the scanner. MR data of thirteen test persons acquired using a new "move during scan" protocol which provides low resolution MR data during the initial movement of the patient bed into the scanner, is used to validate the detection algorithm. When being integrated, our results would enable automatic SAR optimization within the usual acquisition workflow at no extra cost.
\end{abstract}

\section{Introduction}

Recent developments in magnetic resonance imaging (MRI) lead to improvements in the signal-to-noise ratio (SNR) which are especially needed for highresolution and high-speed imaging (e.g., functional imaging). In order to achieve this, the field strength of the static $B_{0}$ field is increased (to $3 \mathrm{~T}$ in current products) which in turn requires higher frequencies for the $B_{1}$ field emitted by the radio frequency $(\mathrm{RF})$ coils. The energy deposition is proportional to the squared $B_{1}$ frequency. Together with dielectric effects occurring at wavelengths close to the dimensions of the human body, this generates more heating of the patient.

This heating is modeled using the specific absorption rate (SAR), given in $\mathrm{W} / \mathrm{kg}$. The international standard [1] requires multiple SAR limits to be complied with (see Section 2.2). They are derived from the requirement of limiting the temperature rise due to RF energy to $1^{\circ}, 2^{\circ}$, and $3^{\circ}$ for head, torso, and extremities, respectively. Staying within these limits is achieved by adjusting the duty cycle (through adjusting repetition time or slice thickness), the flip angle, or the pulse form. If the SAR cannot be estimated accurately, rather large safety margins are required which in turn reduce scan efficiency and/or image quality. 
Addressing this issue requires the development of more accurate monitoring and control algorithms for the RF energy applied to the patient. These algorithms in general have to solve two problems: First, a patient model is needed for simulating SAR values inside humans. Second, for transfering this simulation data to an actual patient for SAR estimation, the positioning of the patient has to be detected. The latter is the topic of the work we are presenting here. Since patients can only be positioned inside a tubular MR scanner in a few different ways (a patient may not lie diagonal or crossways inside the tube), the detection of the positioning effectively reduces to a detection of the patient's axial position with respect to the scanner.

In order to correctly align an SAR model with the actual patient, current systems rely on the manual input of a few patient parameters (usually weight, height, sex, and age) by the doctor. Furthermore, the patient's head has to be positioned onto a given spot on the bed.

There are numerous drawbacks associated with this procedure: Not only is it tedious for the radiology staff but it also takes valuable time from the MRI system. In addition, entering parameters and positioning a patient is error-prone (due to estimation, erroneous weight/height declarations by patients, inexact positioning, and reluctance to enter reasonable values). Therefore, we investigate the possibility of detecting the position relative to the scanner using image processing.

Usually, there are at least two sensors already available for performing such a detection: An optical camera which is used to monitor the patient from the operating room, and the MR imaging device itself. Since the latter is already integrated with the workstation software and new protocols for fast scans (called "move during scan") will be available in products soon, a fast and low-dose prescan will provide the desired input.

\section{Related Work}

\subsection{SAR Estimation}

To our knowledge, so far only [2] explicitly suggested the design of a "smart scan software" for better adjusting scan parameters to individual patients. But of course, the purpose of all the work done on simulating/estimating SAR values is to improve the scan parameter adjustment. These simulations (e.g., [23]) are often based on the Finite Difference Time Domain (FDTD) method and use patient models generated from whole body MR scans like The Visible Human Project ${ }^{\circledR}$ [4]. The following section is based on the results of [5].

\subsection{Critical Limits for SAR}

Table 1 is reproduced from the International Electrotechnical Comission's standard [1, section 51.103.2] and shows the global SAR limits for two different operating modes. This standard also defines rules on which subset of limits has to be obeyed: For exposure with volume RF transmit coils like an integrated 
whole body coil, only the global SAR limits from Table 1 apply. In all other cases (using local RF transmit coils), additional local limits apply. Since position detection is not an issue when using local coils (they are usually positioned accurately), we will concentrate on body coils.

Table 1. Global SAR limits from [1, section 51.103] for an averaging time of $6 \mathrm{~min}$

\begin{tabular}{llll}
\hline & Whole Body & Exposed Body Part & Head \\
\hline Normal Mode & $2 \mathrm{~W} / \mathrm{kg}$ & $2 \mathrm{~W} / \mathrm{kg}-10 \mathrm{~W} / \mathrm{kg}$ & $3.2 \mathrm{~W} / \mathrm{kg}$ \\
First Level Mode & $4 \mathrm{~W} / \mathrm{kg}$ & $4 \mathrm{~W} / \mathrm{kg}-10 \mathrm{~W} / \mathrm{kg}$ & $3.2 \mathrm{~W} / \mathrm{kg}$ \\
\hline
\end{tabular}

${ }^{a}$ Scaling is coupled to the ratio "exposed body mass / total patient mass"

A simulation of the whole body, exposed body part, and head SAR is shown in Fig. 1(a) Although restrictions exist for each of these three values, it is clear that only one of them is a limiting factor to the system. Introducing the SAR-to-limit ratio (STLR) and replotting this graph (see Fig. 1(b) clearly shows that the neck area is crucial, because it defines the transition of the limiting STLR value from head to whole body. See also the symmetry of the energy (proportional to squared field strength) of the limit-adjusted $B_{1}$ field to the head and whole body STLR, respectively. Therefore, we are especially concerned about neck detection. The plot of the adjusted $B_{1}^{2}$ clearly shows that scan performance / image quality is lost in the head and feet sections when setting a flat $B_{1}$ limit (given by the minimal adjusted $B_{1}^{2}$ at the patient's torso).

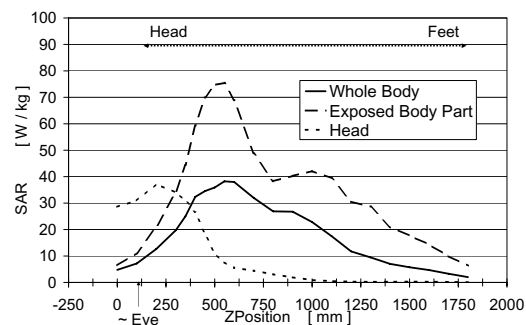

(a) SAR simulation for normal mode at $100 \%$ duty cycle

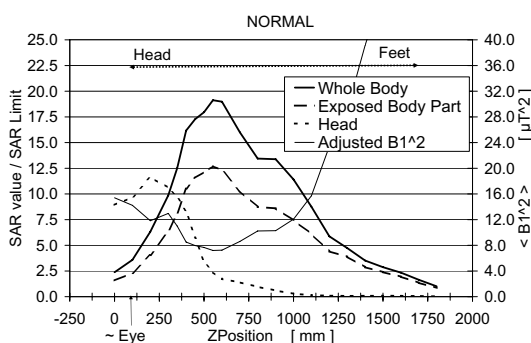

(b) STLR values for normal mode at $100 \%$ duty cycle and adjusted $B_{1}^{2}$

Fig. 1. Simulations for a $3 \mathrm{~T}$ scanner with a reference $B_{1}$ field strength of $11.73 \mu \mathrm{T}$

\section{Methods}

\subsection{Low-Dose Prescan / Move During Scan}

Protocols where the patient bed is moving while a scan is performed are currently developed and are already available for testing our position detection algorithm. The resulting image data comes at no extra cost and is not intended for diagnosis 
as its resolution is quite low. Nevertheless, it is perfectly suited for our application which only requires the data to be good enough for distinguishing different body parts. The image data delivered by MR scans depicts the patient's anatomy, neglecting environment details such as the patient bed. This is probably the biggest advantage compared to other types of sensors. Therefore, an MR scan is a good choice for determining the patient's parameters, if it is fast and does not require an SAR estimation itself. However, these two issues are easily overcome by using a low-dose prescan which is performed during a constant and relatively fast movement of the patient bed with a very low dose. Although the resulting image resolution is quite low (64 by 64 pixels per slice, and a slice spacing of $7.5 \mathrm{~mm}$ to $15 \mathrm{~mm}$ ), the image quality is more than sufficient for estimating the desired parameters. It should be reiterated that the data obtained in this manner is not intended for diagnosis but just for parameter estimation.

\subsection{Position Detection Methods}

We only evaluated slice-based methods, since working on 3D data would restrict the workflow to obtaining a full prescan before starting the actual diagnostic scan. This is time-consuming and therefore needs to be avoided as often as possible. Our goal is to develop an algorithm which only requires prescan data from the body sections that should also be scanned for diagnosis, keeping the workflow as-is. In the following subsections, we will shortly summarize the methods we investigated for detecting the patient's $z$ position relative to the body coil. All these methods are able to make decisions in real time which is required for a smooth workflow.

Area Computations for Thorax and Neck Detection. An obvious measure for classifying slices into body sections is based on slice area computations. The threshold for separating patient from background is easily determined once for all data sets. The derivative of the slice area with respect to the $z$ position (basically the area difference of two successive slices) is a useful measure enabling the localization of the neck section by applying a gradient descent after proper initialization. This initialization is based on the detection of head and thorax that are going to be explained next.

A very robust method for detecting the thorax region is based on the fact that the lungs contain air. This results in the lungs having similar intensities as the background. After thresholding, the lungs build a cavity whose area can be calculated easily: Performing a region growing on the background (using seed points on the slice's top or bottom border), inverting this segmentation, and subtracting the thresholded slice's area yields the desired measure (see Fig. 2). This approach is very robust and is almost impossible to fail in a clinical setting.

Principal Component Analysis. Principal component analysis (PCA), also referred to as Karhunen-Loève expansion, is a well-known method for reducing data's dimension while preserving the most significant information. This is achieved by computing a new data-specific coordinate system so that the first 


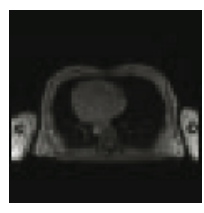

(a) Original

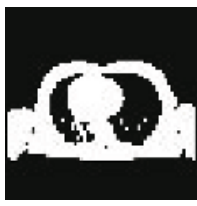

(b) Thresholded

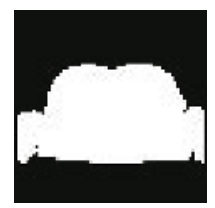

(c) Inverted region growing

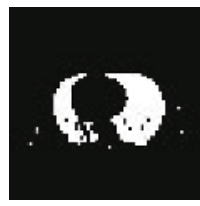

(d) Difference

Fig. 2. Area computations on a $64 \times 64$ thorax slice

few axes / coordinates cover most of the data's variance. PCA originated from the early work of Pearson [6] and was first introduced into statistics by Hotelling 7]. It is used in applications as diverse as model reduction for systems of differential equations and face recognition 89], the latter one being quite related to our problem. Although being a traditional image recognition and representation technique, PCA is still under investigation. E.g., [10] tries to better adopt PCA to $2 \mathrm{D}$ images. In our context, we apply PCA for reducing the image data's dimension before classifying slices $s \in \mathbb{R}^{n^{2}}$ into classes such as "head slice" or "feet slice". This has the two advantages that classification can be done much faster and that irrelevant information is neglected, thereby improving classification accuracy.

For creating a new, variance-specific image basis, the covariance matrix of a large set of representative slices has to be set up. Computing an eigenvalue decomposition

$$
\Sigma B=D B
$$

for the covariance matrix $\Sigma$ (where $D$ is a diagonal matrix containing $\Sigma$ 's eigenvalues in descending order), yields the desired new basis $B \in \mathbb{R}^{n^{2} \times n^{2}}$, whose columns are $\Sigma$ 's eigenvectors. A representation of a (de-meaned) slice image in this new basis is obtained by projecting it onto the new basis $B$. For reducing the data's dimensionality, one may omit eigenvectors corresponding to small eigenvalues and only keep the image's coefficients that correspond to the first few coordinates in the new system. (This corresponds to deleting columns of $B$.) The number of these "principal components" to be kept is to be chosen so that all necessary information is retained, while reducing the set of basis vectors as much as possible. A common method for finding this cut-off is to examine the ratios of successive eigenvalues.

In our case, PCA is employed to detect basic shapes for distinguishing head and feet slices. We downsampled all slices to 16 by 16 pixels in order to accelerate the learning phase. This also enables us to anticipate even faster (and therefore coarser) prescans and to proof the feasibility of PCA for our classification task. There exist various criteria on how many principal components should be kept. This decision has to exhibit a good balance between covered variances, performance of classification, and storage requirements. Extensive testing showed that this is best achieved by reducing the full 256 dimensional image space to approximately 50 principal components. 
We did not apply any normalization of the slices to their centers of gravity for two reasons: First of all, patient movement is very limited in the up/down or left/right direction due to gravity and narrow patient beds, respectively. Furthermore, a translation of slices to their centers of gravity would even degrade the results, since slices depicting a single foot for example would become similar to head slices. A normalization would only be reasonable when being performed on the full $3 \mathrm{D}$ volume, effectively averting the aforementioned problem. But as mentioned at the beginning of this section, we restrict ourselves to slice processing in order to be able to work on partial scans as well.

The actual classification task is then solved by building training sets for the head and feet classes and computing their means $\bar{s}_{\mathrm{H}}$ and $\bar{s}_{\mathrm{F}}$ as well as their covariance matrices $\Sigma_{\mathrm{H}}$ and $\Sigma_{\mathrm{F}}$. A new slice can then be classified by comparing the Mahalanobis distances

$$
d_{\mathrm{H} / \mathrm{F}}(s)=\sqrt{\left(s-\bar{s}_{\mathrm{H} / \mathrm{F}}\right)^{\top} \Sigma_{\mathrm{H} / \mathrm{F}}^{-1}\left(s-\bar{s}_{\mathrm{H} / \mathrm{F}}\right)}
$$

to the means of each of these classes. This is a simple distance classificator with negligible computational cost.

Classification of a patient as "head-first" or "feet-first" is not achieved by evaluating just a single slice, but by accumulating Mahalanobis distances along the $z$ direction until a predefined threshold for this trust value is reached. The accumulation uses the difference of Mahalanobis distances and makes decisions much more robust and reliable. We define the head trust of a partial scan consisting of slices $s_{1}, \ldots, s_{k}$ as

$$
T_{\mathrm{H}}(k)=\sum_{i=1}^{k} t_{\mathrm{H}}\left(s_{i}\right), \quad \text { with } \quad t_{\mathrm{H}}(s)= \begin{cases}d_{\mathrm{H}}^{-1}(s), & \text { if } d_{\mathrm{F}}(s)>d_{\mathrm{H}}(s)+\Delta . \\ 0, & \text { otherwise }\end{cases}
$$

The feet trust is defined analoguously. In empirical studies, a difference threshold of $\Delta=10$ showed good results.

\section{Experiments and Results}

\subsection{Image Data}

We acquired whole body scans of 13 test persons. The subjects were positioned in different ways. Some were instructed to put a pillow below their feet and some put their head left of the patient bed's center line. The resolution used for acquiring the images was $7.5 \mathrm{~mm}$ in $x$ and $y$ direction and $7.5 \mathrm{~mm}$ to $15 \mathrm{~mm}$ in $z$ direction. Each slice originally had 64 by 64 pixels. It has to be reiterated that, before applying PCA, all images were downsampled in $x$ and $y$ direction to 16 by 16 pixels, yielding a resolution of $3 \mathrm{~cm}$.

\subsection{Detection Results for Crucial Sections of the Body}

In this section, we will summarize the results of our experiments to detect several sections of the human body. For all detections, we had to define thresholds for 
the corresponding measures. These thresholds were chosen by experience once for all datasets.

Head and Feet. For testing the PCA classification, the 13 data sets were divided into 4 learning sets and 9 test sets. After downsampling in $x$ and $y$ direction, applying PCA, and transforming the images into the new space, slices were collected for every class to be trained. After this supervised learning phase, the classification of a data set into "head-first" or "feet-first" was done using the trust values defined in (2). We were able to robustly detect the head and feet of all patients in the test set by making a decision only if one of the two trust values reached a predefined threshold while the other one remained zero. Even persons with a pillow below their feet or with their head not center-positioned were accurately classified, since one of the four training data sets shared these properties. See Figs. 3(a) 3(c) for exemplary distances and trust values of one data set.

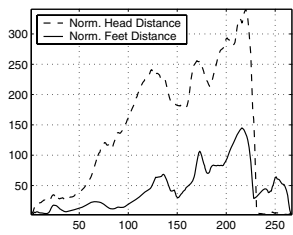

(a)

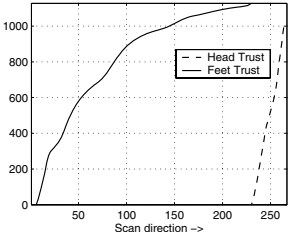

(b)

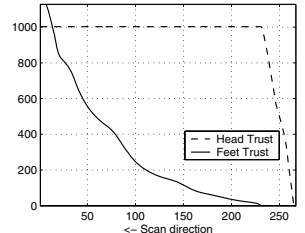

(c)

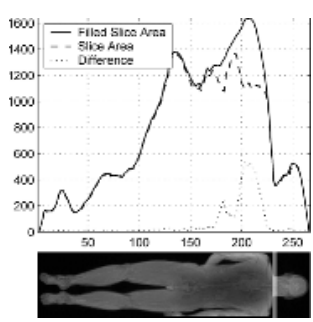

(d)

Fig. 3. Figs. (a) (c) show the normalized Mahalanobis distances and the trust values for head / feet first classification. Fig. (d) shows the area measures used for detecting the same patient's thorax and neck. The $x$ axis corresponds to the slice number.

Thorax. The area comparison described above works very robust and fast in a clinical setting. Since every patient has lungs, the only requirement is that they form a cavity. This is guaranteed unless the patient's chest is opened during an intervention. However, for testing, we did not instruct the subjects to wear scrubs (as this is usually done in clinical routine) instead of their own clothing. This resulted in artifacts from metal buttons or zips. These artifacts destroyed the topology by canceling the signal in two data sets. In all other data sets the thorax was detected accurately. See Fig. $3(\mathrm{~d})$ for the area measures for one data set.

Neck. As stated in section 2.2, the detection of the patient's neck is crucial since in this body section the confining SAR limit changes from the head limit to one of the other two limits (whole body or exposed body part, depending on the operation mode). Our approach for detecting the neck is based on a combination of three methods: The slice classification for detecting the head position, the region growing on background for detecting the thorax, and a combined 
area / area gradient analysis for determining the neck slices between head and thorax (see Fig. 3(d)]. As long as head and thorax are detected, neck detection works perfectly.

\section{Discussion}

Currently, we are working on using a broader range of criteria to base our decisions on. This leads us to the addition of a classification system (e.g., a naive Bayes classifier would probably do the job) at the end of slice processing in order to robustly combine more features than just PCA, area difference, and area gradient. This modification may also empower us to detect more body parts than just head, neck, and feet and additionally would be more robust (regarding image artifacts). After that, the detection algorithm could be integrated within the duty cycle management software of MR scanners.

\section{Conclusion}

In this paper, we described the importance of SAR optimization for a smooth and safe MRI acquisition. Currently, MR imaging requires manual input from the radiology staff and is prone to different errors and omissions. We propose an automatic solution based on the fast and low resolution "move during scan" protocol. The detection and classification methods used provide a simple and robust solution for the detection of patient position and the location of the patient's neck which is crucial for SAR planning. Results on thirteen test persons validate the approach.

This paper presents, to the best knowledge of the authors, the first attempt to automatically detect the patient's position with respect to the MR scanner and, therefore, enables a new solution towards designing a safe, fast, and smooth workflow for magnetic resonance image acquisition.

\section{References}

1. International Electrotechnical Comission: (IEC 60601 medical electrical equipment - part 2-33)

2. Zhai, Z., DeMeester, G.D., Morich, M.A., Harvey, P.R., Kleihorst, R.P.: Optimization of transmit efficiency for a $\mathrm{T} / \mathrm{R}$ whole body coil at $3 \mathrm{~T}$. In: Proc. Int'l Soc. Mag. Reson. Med. Volume 13. (2005)

3. Zhai, Z., DeMeester, G.D., Shvartsman, S., Morich, M.A.: FDTD calculations of $\mathrm{B}_{1}$-field and SAR for $3 \mathrm{~T}$ whole body coil. In: Proc. Int'l Soc. Mag. Reson. Med. Volume 10. (2002)

4. Ackerman, M.J.: The visible human project. Proc. of the IEEE 86(3) (1998) 504-511 National Library of Medicine, Bethesda, MD.

5. Diehl, D., Weinert, U., Röckelein, R., Brinker, G.: SAR-calculations of a realistic, scalable human model in a MRI-resonator. In: Proc. Int'l IGTE Symposium on Numerical Field Calculation in Electrical Engineering. (2002) 
6. Pearson, K.: On lines and planes of closest fit to systems of points in space. Philosophical Mag. (1901)

7. Hotelling, H.: Analysis of a complex of statistical variables into principal components. J. of Educational Psychology 24 (1933) 417-441, 498-520

8. Kirby, M., Sirovich, L.: Application of the Karhunen-Loève procedure for the characterization of human faces. IEEE Trans. PAMI 12(1) (1990) 103-108

9. Turk, M.A., Pentland, A.P.: Face recognition using eigenfaces. In: Proc. IEEE Conf. Computer Vision and Pattern Recognition (CVPR). (1991) 586-591

10. Yang, J., Zhang, D., Frangi, A.F., Yang, J.y.: Two-dimensional PCA: A new approach to appearance-based face representation and face recognition. IEEE Trans. PAMI 26(1) (2004) 131-137 